\title{
SCALING MATRICES TO PRESCRIBED ROW AND COLUMN MAXIMA*
}

\author{
URIEL G. ROTHBLUM $\dagger$, HANS SCHNEIDER $\ddagger$ AND MICHAEL H. SCHNEIDER§
}

\begin{abstract}
A nonnegative symmetric matrix $B$ has row maxima prescribed by a given vector $r$, if for each index $i$, the maximum entry in the $i$ th row of $B$ equals $r_{i}$. This paper presents necessary and sufficient conditions so that for a given nonnegative symmetric matrix $A$ and positive vector $r$ there exists a positive diagonal matrix $D$ such that $B=D A D$ has row maxima prescribed by $r$. Further, an algorithm is described that either finds such a matrix $D$ or shows that no such matrix exists. The algorithm requires $O(n \lg n+p)$ comparisons, $O(p)$ multiplications and divisions, and $O(q)$ square root calculations where $n$ is the order of the matrix, $p$ is the number of its nonzero elements, and $q$ is the number of its nonzero diagonal elements. The solvability conditions are compared and contrasted with known solvability conditions for the analogous problem with respect to row sums. The results are applied to solve the problem of determining for a given nonnegative rectangular matrix $A$ positive, diagonal matrices $D$ and $E$ such that $D A E$ has prescribed row and column maxima. The paper presents an equivalent graph formulation of the problem. The results are compared to analogous results for scaling a nonnegative matrix to have prescribed row and column sums and are extended to the problem of determining a matrix whose rows have prescribed $l_{p}$ norms.
\end{abstract}

Key words. symmetric matrices, potential, directed graph, weighted graph, scalings, row-maxima

AMS subject classifications. 05L20, 15A99, 90B10

1. Introduction. A matrix $B$ is called a symmetric scaling of a nonnegative square matrix $A$ if $B=D A D$ for some positive diagonal matrix $D$. A matrix $B$ is called an equivalence scaling of a nonnegative rectangular matrix $A$ if $B=D A E$ for some positive diagonal matrices $D$ and $E$. In this paper we give necessary and sufficient conditions that a given symmetric nonnegative matrix $A$ has a symmetric scaling with prescribed row maxima. In particular, we show that for a given pattern (i.e., locations of strictly positive entries) if the class of symmetric nonnegative matrices with that pattern and having the prescribed row maxima is nonempty, then every nonnegative matrix with that pattern can be symmetrically scaled into the class. Thus, our conditions relate the prescribed row maxima to the pattern of the matrix $A$.

Further, we describe an algorithm that for a given matrix $A$ either determines a symmetric scaling $B$ with prescribed row maxima or shows that no such scaling exists. Using our results for symmetric scalings, we also establish corresponding results for the problem of determining an equivalence scaling of a rectangular nonnegative matrix with prescribed row and column maxima. Our results have natural interpretations in terms of weighted undirected graphs.

We call the problem of finding for a given square nonnegative matrix a symmetric scaling with prescribed row maxima max symmetric scaling and the problem of finding for a given rectangular nonnegative matrix an equivalence scaling with prescribed row

* Received by the editor November 19, 1991; accepted for publication (in revised form) May 11, 1992.

$\dagger$ Rutgers Center for Operations Research, Rutgers University, New Brunswick, New Jersey 08903 and Faculty of Industrial Engineering and Management, Technion-Israel Institute of Technology, Technion City, Haifa 32000, ]srael (IERUR10@TECHNION). Research supported in part by Israel-United States Binational Science Foundation grant 87-00194.

$\ddagger$ Department of Mathematics, University of Wisconsin, Madison, Wisconsin 53706 (hans@!math.wisc.edu). Research supported in part by National Science Foundation grants DMS-8901445 and ECS 87-18971, Binational Science Foundation grant 87-00194, and the International Scientific Research Program, the Ministry of Education, Science and Culture, Japan.

§ AT\&T Bell Laboratories, HO 3K-316, Holmdel, New Jersey 07733 (mschneider@attmail.com ) and Department of Mathematical Sciences, Johns Hopkins University, Baltimore, Maryland 21218. Research supported in part by National Science Foundation grant ECS 87-18971. 
and column maxima max equivalence scaling. These problems are analogues of corresponding well-studied scaling problems in which row and column sums are prescribed. We refer to the sum versions of these problems as sum symmetric scaling and sum equivalence scaling. The problem of sum equivalence scaling was described in the engineering literature by Kruithof [10] and was considered by Sinkhorn [17], Brualdi [5], Sinkhorn and Knopp [18], Bregman [1], Menon [12], Menon and Schneider [13], Schneider and Zenios [16], and many other authors. The sum symmetric problem was considered by Brualdi [3], [4] and Marshall and Olkin [11].

One important application of sum equivalence scaling concerns the updating of (dynamic) data that is given in matrix form, e.g., traffic intensity between sources and destinations. When new data is not fully observable, but new marginals consisting of corresponding row sums and column sums are observable, a common technique is to replace the old data given by a matrix $A$ by a scaling $D A E$ whose row sums and column sums equal the observed marginals. Max equivalence scaling arises naturally when observations about the new data concern row and column maxima.

We describe our notation in $\S 2$ and list some solvability results for sum symmetric and sum equivalence scalings in $\S 3$. We consider these problems both for a given pattern and for all subpatterns of a given pattern, and we add some new results in the latter case. In $\S 4$ we give nine equivalent conditions for the existence of a solution of the max symmetric scaling. In $\S 5$ we present an algorithm that, for a given nonnegative matrix $A$, either symmetrically scales $A$ to have prescribed row maxima or determines that no such scaling can exist. In $\S 6$, we apply the results of $\S 4$ to study max equivalence scaling, and in $\S 7$ we restate our results in terms of weighted undirected graphs. Finally, in $\S 8$ we unify max symmetric scaling and sum symmetric scaling by considering scaling problems in which the $l_{p}$ norms of the rows of the matrix are prescribed.

2. Notation and definitions. For a positive integer $n$, we use the notation $\langle n\rangle$ to denote the set of integers $\{1,2, \ldots, n\}$. For a subset $I \subseteq\langle n\rangle$, we use $I^{c}$ to denote the set $\langle n\rangle \backslash I$, the complement of $I$ with respet to $\langle n\rangle$. The identity of $n$ will always be clear from the context. The cardinality of a finite set $S$ is denoted $|S|$. Also, we use the symbols $\subset$ and $\subseteq$ to denote strict and weak containment, respectively.

Let $A$ be an $m \times n$ nonnegative matrix and let $I$ and $J$ be nonempty subsets of $\langle m\rangle$ and $\langle n\rangle$, respectively. We use the notation $A_{I J}$ to denote the $|I| \times|J|$ submatrix of $A$ corresponding to the rows and columns of $A$, indexed by $I$ and $J$, respectively. We identify an index $i$ and the set $\{i\}$. For example, when $I=\{i\}$, we write $A_{i J}$ for $A_{I J}$. By convention, we write $A_{I J}=0$ if either $I$ or $J$ equals the empty set.

For a vector $r=\left(r_{1}, \ldots, r_{n}\right)^{T} \in \Re^{n}$ and subset $I \subseteq\langle n\rangle$, we use the notation $r_{I}$ to denote the subvector of $r$ whose entries are $r_{i}$ for $i \in I$, and we use $r(I)$ to denote the element sum of $r_{I}$. We follow the standard convention that the summation over the empty set is defined to be 0 . Also, the value of $\max _{i \in I} r_{i}$ in the case of $I=\varnothing$ depends on the underlying group to which the elements $r_{i}$ belong. Specifically, if we are considering the entries of $r$ as entries of the multiplicative group of nonnegative real numbers, then the maximization over the empty set is defined to be 0 , whereas if the entries are viewed as elements of the additive group of real numbers, then the maximization over the empty set is defined to be $-\infty$. The additive case arises in $\S 7$ when we consider graph versions of our results. Also, for $\alpha>0$, we define the operation $\frac{\alpha}{0}=+\infty$. This operation will occur only in minimization expressions over sets containing an element of finite value.

The $n \times n$ diagonal matrix whose diagonal entries are $d_{1}, d_{2}, \ldots, d_{n}$ is denoted $\operatorname{diag}\left(d_{1}, d_{2}, \ldots, d_{n}\right)$. A diagonal matrix $D=\operatorname{diag}\left(d_{1}, d_{2}, \ldots, d_{n}\right)$ is called positive if $d_{i}>0$ for $i \in\langle n\rangle$. For an $n \times n$ nonnegative symmetric matrix $A$, a matrix $B$ is called 
a symmetric scaling of $A$ if $B=D A D$ for some positive diagonal matrix $D$. For an $m \times n$ nonnegative matrix $A$, a matrix $B$ is called an equivalence scaling of $A$ if $B=$ $D A E$ for some positive diagonal matrices $D$ and $E$.

An $m \times n$ matrix $P=\left[p_{i j}\right]$ is called a pattern matrix if every entry of $P$ is either 0 or 1. Given two $m \times n$ pattern matrices $P$ and $P^{\prime}$, the matrix $P^{\prime}$ is a subpattern of $P$ if $P^{\prime} \leqq P$. Given a $m \times n$ nonnegative matrix $A=\left[a_{i j}\right]$, the pattern of $A$ is the $m \times n$ pattern matrix $P$ such that for $i \in\langle m\rangle$ and $j \in\langle n\rangle$

$$
p_{i j}=\left\{\begin{array}{ll}
1 & \text { if } a_{i j}>0, \\
0 & \text { otherwise }
\end{array}\right. \text { and }
$$

For an $m \times n$ pattern matrix $P$, we define the pattern class of $P$, written $\Pi(P)$, to be the set of all $m \times n$ nonnegative matrices whose pattern is $P$.

3. Existence conditions for sum scaling. We summarize numerous characterizations for the solvability of sum symmetric scaling and sum equivalence scaling.

We call a matrix, or a vector, positive if all of its elements are positive. For a positive vector $r=\left(r_{i}, \ldots, r_{n}\right)^{T} \in \Re^{n}$, let $S(r)$ denote the set of all $n \times n$ nonnegative matrices $A=\left[a_{i j}\right]$ such that

$$
\sum_{j=1}^{n} a_{i j}=r_{i} \text { for } i \in\langle n\rangle .
$$

Conditions (i), (ii), (iii), and (vi) of the following theorem are contained in Brualdi [3], [4]. Conditions (iv) and (v) are, apparently, new.

THEOREM 1. Let $P$ be an $n \times n$ symmetric pattern matrix, and let $r \in \Re^{n}$ be strictly positive. Then the following are equivalent:

(i) Each symmetric $A \in \Pi(P)$ has a symmetric scaling $B$ in $S(r)$.

(ii) Some symmetric $A \in \Pi(P)$ has a symmetric scaling $B$ in $S(r)$.

(iii) The set $\Pi(P) \cap S(r)$ is nonempty.

(iv) If $I$ and $J$ are subsets of $\langle n\rangle$ such that $P_{I J}=0$, then $r(I) \leqq r\left(J^{c}\right)$ with equality holding if and only if $P_{I^{c} J^{c}}=0$.

(v) If $I$ and $J$ are subsets of $\langle n\rangle$ such that $P_{I J}=0$, then $r(I \cap J) \leqq r\left((I \cup J)^{c}\right)$ with equality holding if and only if $P_{(I \cap J)^{c},(I \cup J)^{c}}=0$.

(vi) If $\{K, L, M\}$ is any partition of $\langle n\rangle$ such that $P_{K, K \cup L}=0$, then $r(K) \leqq r(M)$ with equality holding if and only if $P_{L \cup M, M}=0$.

Proof. The equivalence of (i), (ii), (iii), and (vi) is given in Brualdi [3], [4]. The implication (iii) $\Rightarrow$ (iv) is found in [13], and the equivalence of (iv) and (v) follows from the observations that $r(I)=r(I \cap J)+r(I \backslash J)$ and $r\left(J^{c}\right)=r\left((I \cup J)^{c}\right)+r(I \backslash J)$. Finally, to see that $(\mathrm{v}) \Rightarrow$ (vi), consider a partition $\{K, L, M\}$ of $\langle n\rangle$ such that $P_{K, K \cup L}=0$. Then apply (v) to the sets $K$ and $K \cup L$.

For positive vectors $r=\left(r_{1}, \ldots, r_{m}\right)^{T} \in \Re^{m}$ and $c=\left(c_{1}, \ldots, c_{n}\right)^{T} \in \Re^{n}$, let $S(r, c)$ be the set of all $m \times n$ nonnegative matrices $A=\left[a_{i j}\right]$ such that

$$
\sum_{j=1}^{n} a_{i j}=r_{i} \quad \text { for } i \in\langle m\rangle \text { and } \sum_{i=1}^{m} a_{i j}=c_{i} \quad \text { for } j \in\langle n\rangle .
$$

The following theorem summarizes results of Menon [12], Brualdi [2], and Menon and Schneider [13].

THEOREM 2. Let $P$ be an $m \times n$ pattern matrix, and let $r \in \Re^{n}$ and $c \in \Re^{m}$ be strictly positive. Then the following are equivalent:

(i) Each matrix $A \in \Pi(P)$ has an equivalence scaling $B$ in $S(r, c)$.

(ii) Some matrix $A \in \Pi(P)$ has an equivalence scaling $B$ in $S(r, c)$. 
(iii) The set $\Pi(P) \cap S(r, c)$ is nonempty.

(iv) If $I$ and $J$ are subsets of $\langle m\rangle$ and $\langle n\rangle$, respectively, such that $P_{I J}=0$, then $r(I) \leqq c\left(J^{c}\right)$ with equality holding if and only if $P_{I^{c}, J^{c}}=0$.

The following two theorems characterize solvability of sum equivalence scaling and sum symmetric scaling for subpatterns of a given pattern matrix $P$, respectively.

THEOREM 3. Let $P$ be an $m \times n$ pattern matrix, and let $r \in \Re^{m}$ and $c \in \Re^{n}$ be strictly positive. Then the following are equivalent:

(i) For some subpattern $P^{\prime}$ of $P$, each $A \in \Pi\left(P^{\prime}\right)$ has an equivalence scaling in $S(r, c)$.

(ii) For some subpattern $P^{\prime}$ of $P$, some matrix $A \in \Pi\left(P^{\prime}\right)$ has an equivalence scaling in $S(r, c)$.

(iii) For some subpattern $P^{\prime}$ of $P$, the set $\Pi\left(P^{\prime}\right) \cap S(r, c)$ is nonempty.

(iv) If $I$ and $J$ are subsets of $\langle m\rangle$ and $\langle n\rangle$, respectively, such that $P_{I J}=0$, then $r(I) \leqq c\left(J^{c}\right)$.

Proof. The equivalence of (i), (ii), and (iii) is immediate from Theorem 2 applied to a corresponding subpattern $P^{\prime}$ of $P$. A proof of the equivalence of (iii) and (iv) of Theorem 3, which is simpler than the one given in [14], can be found in [15].

THEOREM 4. Let $P$ be an $n \times n$ symmetric pattern matrix, and let $r \in \Re^{n}$ be strictly positive. Then the following are equivalent:

(i) For some symmetric subpattern $P^{\prime}$ of $P$, each symmetric $A \in \Pi\left(P^{\prime}\right)$ has a symmetric scaling in $S(r, c)$.

(ii) For some symmetric subpattern $P^{\prime}$ of $P$, some symmetric $A \in \Pi\left(P^{\prime}\right)$ has a symmetric scaling in $S(r, c)$.

(iii) For some symmetric subpattern $P^{\prime}$ of $P$, the set $\Pi\left(P^{\prime}\right) \cap S(r)$ is nonempty.

(iv) If $I$ and $J$ are subsets of $\langle n\rangle$ such that $P_{I J}=0$, then $r(I) \leqq r\left(J^{c}\right)$.

(v) If $I$ and $J$ are subsets of $\langle n\rangle$ such that $P_{I J}=0$, then $r(I \cap J) \leqq r\left((I \cup J)^{c}\right)$.

(vi) If $\{K, L, M\}$ is any partition of $\langle n\rangle$ such that $P_{K, K \cup L}=0$, then $r(K) \leqq r(M)$.

Proof. The equivalence of (i), (ii), and (iii) follows directly from Theorem 1, applied to a corresponding subpattern $P^{\prime}$ of $P$.

(iii) $\Rightarrow$ (iv): If (iii) is satisfied for subpattern $P^{\prime}$ of $P$ and $I, J \subseteq\langle n\rangle$ with $P_{I J}=0$, then $P_{I J}^{\prime}=0$, and it follows from the implication (iii) $\Rightarrow$ (iv) of Theorem 1 that $r(I) \leqq$ $r\left(J^{c}\right)$, and therefore (iv) holds.

(iv) $\Leftrightarrow$ (v) $\Rightarrow$ (vi): These implications follow from the arguments used to show the analogous implications of Theorem 1 .

(vi) $\Rightarrow$ (v): Assume that (vi) holds and that $I, J \subseteq\langle n\rangle$ with $P_{I J}=0$. Then $P_{I \cap J, J}=0$, and by the symmetry of $P$ we have $P_{I \cap J, I \backslash J}=\left[P_{I \backslash J, I \cap J}\right]^{T}=0$; therefore, $P_{I \cap J, I \cup J}=0$. Applying condition (vi) to the partition $\{K, L, M\}$ with $K=I \cap J$, $L=(I \backslash J) \cup(J \backslash I)$, and $M=(I \cup J)^{c}$, it follows that $r(I \cap J)=r(K) \leqq r(M)=$ $r\left((I \cup J)^{c}\right)$.

(iv) $\Rightarrow$ (iii): We prove this implication using a modification of the technique used in the proof of Theorem 3.7 in [4]. Suppose that (iv) holds. It follows from the implication (iv) $\Rightarrow$ (iii) of Theorem 3 with $m=n$ and $c=r$ that for some subpattern $Q$ (which need not be symmetric) of $P$ there exists a matrix $C \in \Pi(Q)$ satisfying (2) with $c=r$. Then $B=\frac{1}{2}\left(C+C^{T}\right)$ satisfies (1) and $B \in \Pi\left(P^{\prime}\right)$, where $P^{\prime}=\frac{1}{2}\left(Q^{\prime}+Q^{T}\right)$ is a symmetric subpattern of $P$.

We observe that it suffices to prove that for some subpattern $P^{\prime}$ (which need not be symmetric) of $P$ there exists a matrix $B \in \Pi\left(P^{\prime}\right)$ satisfying (2) with $c=r$ (and, consequently, $m=n)$. This follows because if $B$ is such a matrix, then $B^{\prime}=\frac{1}{2}\left(B+B^{T}\right)$ satisfies (1) and $B^{\prime} \in \Pi(Q)$, where $Q=\frac{1}{2}\left(P^{\prime}+\left(P^{\prime}\right)^{T}\right)$ is a symmetric subpattern of $P$. Therefore, the implication follows from the implication (iv) $\Rightarrow$ (iii) of Theorem 3 . 
4. Problem statement and existence conditions. We present our main result giving eight equivalent conditions characterizing the existence of a solution for max symmetric scaling.

For a positive vector $r=\left(r_{1}, \ldots, r_{n}\right)^{T} \in \Re^{n}$, let $M(r)$ denote the set of all $n \times n$ nonnegative matrices $A=\left[a_{i j}\right]$ such that

$$
\max _{j \in\langle n\rangle} a_{i j}=r_{i} \text { for } i \in\langle n\rangle \text {. }
$$

Also for a vector $r \in \Re^{n}$ and scalar $\alpha \in \Re$, let the $\alpha$-level set of $r$, denoted lev $(r, \alpha)$, be the set $\left\{i \in\langle n\rangle: r_{i} \geqq \alpha\right\}$. Finally, if we have that $A$ is an $n \times n$ symmetric matrix and $I=\operatorname{lev}(r, \alpha) \neq \phi$, we call $A_{I I}$ an $r$-upper principal submatrix of $A$.

THEOREM 5. Let $P$ be an $n \times n$ symmetric pattern matrix, and let $r \in \Re^{n}$ be strictly positive. Then the following are equivalent:

(i) Each symmetric $A \in \Pi(P)$ has a symmetric scaling in $M(r)$.

(ii) Some symmetric $A \in \Pi(P)$ has a symmetric scaling in $M(r)$.

(iii) The set $\Pi(P) \cap M(r)$ is nonempty. $P^{\prime} \leqq P$.

(iv) The set $\Pi\left(P^{\prime}\right) \cap M(r)$ is nonempty for some pattern matrix $P^{\prime}$ satisfying

(v) If $P_{I J}=0$ for subsets $I, J \subseteq\langle n\rangle$, then

$$
\max _{i \in I} r_{i} \leqq \max _{j \in J^{c}} r_{j}
$$

(vi) If $P_{I J}=0$ for subsets $I, J \subseteq\langle n\rangle$, then

$$
\max _{i \in I \cap J} r_{i} \leqq \max _{j \in(I \cup J)^{c}} r_{j} .
$$

(vii) If $\{K, L, M\}$ is any partition of $\langle n\rangle$ such that $P_{K, K \cup L}=0$, then

$$
\max _{i \in K} r_{i} \leqq \max _{i \in M} r_{i} \text {. }
$$

(viii) If $P_{i J}=0$ for $J \subseteq\langle n\rangle$ and $i \in J$, then

$$
r_{i} \leqq \max _{j \in J^{c}} r_{j}
$$

(ix) No upper r-principal submatrix of $P$ has a zero row.

Proof. The implication (i) $\Rightarrow$ (ii) is trivial because $\Pi(P)$ is nonempty $(P \in \Pi(P))$, and the implication (ii) $\Rightarrow$ (iii) is straightforward because $D A D \in \Pi(P)$ whenever $A \in$ $\Pi(P)$ and $D$ is a positive diagonal matrix. Also, the implication (iii) $\Rightarrow$ (iv) is trivial.

(iv) $\Rightarrow$ (v): Let $A \in \Pi\left(P^{\prime}\right) \cap M(r)$ for some pattern matrix $P^{\prime} \leqq P$, and let $I$ and $J$ be nonempty subsets of $\langle n\rangle$ such that $P_{I J}=0$, and therefore $P_{I J}^{\prime}=A_{I J}=0$. Because $r_{i}$ is the maximum of the entries in the $i$ th row and $A$ is symmetric, it follows directly that

$$
\max _{i \in I} r_{i}=\max _{i \in I} \max _{j \in\langle n\rangle} a_{i j}=\max _{i \in I} \max _{j \in J^{c}} a_{i j}=\max _{j \in J^{c}} \max _{i \in I} a_{i j} \leqq \max _{j \in J^{c}} r_{j} .
$$

(v) $\Rightarrow$ (vi): Let $I$ and $J$ satisfy the assumptions of condition (vi). Then $P_{I \cap J, I \cup J}=$ 0 ( see the proof of (vi) $\Rightarrow(\mathrm{v})$ in Theorem 4 ) and (5) follows by applying (4) to the sets $I \cap J$ and $I \cup J$.

(vi) $\Rightarrow$ (vii): If $K, L$, and $M$ satisfy the assumptions of condition (vii), (6) follows by applying (5) to the sets $I=K$ and $J=K \cup L$ and observing that $K \cap(K \cup L)=K$ and $[K \cup(K \cup L)]^{c}=M$.

(vii) $\Rightarrow$ (viii): If $i$ and $J$ satisfy the assumption of condition (vii), then $\left\{\{i\}, J \backslash\{i\}, J^{c}\right\}$ is a partition of $\langle n\rangle$ satisfying the assumptions of condition (viii), and (7) follows by applying (6). 
(viii) $\Rightarrow$ (ix): Suppose that for some $\alpha \in \Re$ and $J=\left\{j \in\langle n\rangle \mid r_{j} \geqq \alpha\right\} \neq \varnothing$, the $i$ th row of the upper principal submatrix $P_{J J}$ is zero. Then, $i \in J \subseteq\langle n\rangle, P_{i J}=0$, and $r_{i} \geqq$ $\alpha>r_{j}$ for $j \in J^{c}$. This violates condition (viii) for the partition $\left\{\{i\}, J \backslash\{i\}, J^{c}\right\}$ and therefore proves the implication.

(ix) $\Rightarrow$ (i): We prove this implication constructively in $\S 5$ by exhibiting an algorithm that for a given nonnegative symmetric matrix $A$ and positive vector $r$ either finds a symmetric scaling of $A$ in $M(r)$ or finds an upper principal submatrix of $A$ containing a zero row.

The following observations compare max symmetric scaling and sum symmetric scaling. First, note that feasibility conditions (iii) and (iv) in Theorem 5 are equivalent. By contrast, there is no such equivalence for sum symmetric scaling, and the assertion that for some subpattern $P^{\prime}$ of $P$ there is a matrix $A$ in $\Pi\left(P^{\prime}\right)$ satisfying $(1)$ is not equivalent to condition (iii) of Theorem 1. In fact Rothblum and Schneider [14] provide separate characterizations of each of these two conditions. As a result of the equivalence of conditions (iii) and (iv) of Theorem 5, conditions (v), (vi), and (vii) of Theorem 5 are simpler than the analogous conditions (iv), (v), and (vi) of Theorem 1. For example, for the nonequivalence for sum scaling consider

$$
P=\left(\begin{array}{ll}
1 & 1 \\
1 & 0
\end{array}\right) \text { and } r=\left(\begin{array}{l}
1 \\
1
\end{array}\right) \text {. }
$$

Then $P \in M(r)$, but there is no symmetric scaling of $P$ whose rows sums are $(1,1)^{T}$.

Second, as a consequence of properties of the max operation, the set conditions (v), (vi), and (vii) of Theorem 5 are equivalent to the simple point conditions (vii) and (viii). No analogous simplification is possible for sum symmetric scaling.

Third, a solution for sum symmetric scaling is unique, whereas a solution for max symmetric scaling need not be unique. For example, let

$$
A=\left(\begin{array}{ll}
1 & 2 \\
2 & 1
\end{array}\right) \text { and } r=\left(\begin{array}{l}
1 \\
1
\end{array}\right)
$$

Then the general symmetric scaling of $A$ that is in $M(r)$ is given by

$$
B=\left(\begin{array}{cc}
\alpha & 0 \\
0 & (2 \alpha)^{-1}
\end{array}\right) A\left(\begin{array}{cc}
\alpha & 0 \\
0 & (2 \alpha)^{-1}
\end{array}\right)=\left(\begin{array}{cc}
\alpha^{2} & 1 \\
1 & \left(4 \alpha^{2}\right)^{-1}
\end{array}\right),
$$

where $\frac{1}{2} \leqq \alpha \leqq 1$.

5. The algorithm. We describe an algorithm for max symmetric scaling. For a given nonnegative symmetric matrix $A$ and strictly positive vector $r$, our algorithm either finds a symmetric scaling of $A$ in $M(r)$ or certifies that no such scaling exists by showing that condition (ix) of Theorem 5 is violated.

\section{The MaX Symmetric SCALING Algorithm}

Input: An $n \times n$ nonnegative symmetric matrix $A$ and a strictly positive vector $r \in \Re^{n}$.

Output: Either a positive diagonal matrix $D$ such that $D A D \in M(r)$ or a subset $J \subseteq\langle n\rangle$ and an index $i \in J$ such that $A_{J J}$ is an $r$-upper principal submatrix of $A$ with zero row $A_{i J}$.

Step 0 (Initialization): Let $\alpha_{i}$ for $i \in\langle m\rangle$ be the distinct values in the vector $r$ listed in decreasing order. That is,

$$
\left\{\alpha_{i} \mid i \in\langle m\rangle\right\}=\left\{r_{i} \mid i \in\langle n\rangle\right\} \quad \text { and } \quad \alpha_{1}>\alpha_{2}>\cdots>\alpha_{m} .
$$

Set $k=1, J=\varnothing$, and $d_{i}=1$ for $i=1,2, \ldots, n$. 
Step 1 (Push down): Set

$$
I=\left\{i \in\langle n\rangle \mid r_{i}=\alpha_{k}\right\} \quad \text { and } \quad J=\left\{i \in\langle n\rangle \mid r_{i} \geqq \alpha_{k}\right\} .
$$

If $A_{i j}=0$ for some $i \in I$, output $(J, i)$ and STOP; otherwise, set the values $d_{i}$ for $i \in I$ so that $d_{i} \leqq 1$ and

$$
d_{i} a_{i j} d_{j} \leqq \alpha_{k} \text { for } i \in I \text { and } j \in J
$$

Step 2 (Pull up): Select any $i \in I$ and set $I=I \backslash i$. Set

$$
d_{i}=\min \left\{\min _{\substack{j \in J \\ j \neq i}}\left\{\frac{\alpha_{k}}{a_{i j} d_{j}}\right\}, \sqrt{\frac{\alpha_{k}}{a_{i i}}}\right\} .
$$

If $I \neq \varnothing$, repeat Step 2 .

Step 3 (Termination): If $k<m$, replace $k$ by $k+1$ and return to Step 1; if $k=m$, then output $D=\operatorname{diag}\left(d_{1}, d_{2}, \ldots, d_{n}\right)$ and STOP.

We observe that whenever the algorithm does not stop in Step 1, then $A_{I J}$ has no zero row and we can achieve ( 9 ) by setting

$$
d_{i}=\min \left\{\min _{j \in J}\left\{\frac{\alpha_{k}}{a_{i j}}\right\}, 1\right\} \quad \text { for } i \in I .
$$

The following lemma is crucial for our analysis.

LEMMA 6. During the Max Symmetric Scaling Algorithm, after each execution of Step 2, we have

$$
d_{i} a_{i j} d_{j} \leqq r_{i} \quad \text { for } i, j \in J
$$

and

$$
\max _{j \in J} d_{i} a_{i j} d_{j}=r_{i} \quad \text { for } i \in J \backslash I \text {. }
$$

Furthermore, the d's are nondecreasing throughout consecutive executions of Step 2.

Proof. We first show that the $d$ 's are nondecreasing and that if (12) and (13) hold at the beginning of an execution of Step 2, then they also hold at the end of that execution. Let $s$ be the element selected out of $I$ for the execution of Step 2, let $d^{\prime}$ and $I^{\prime}$ be, respectively, the values of $d$ and $I$ at the beginning of the execution of the current Step 2 , and let $d^{\prime \prime}$ and $I^{\prime \prime}$ be the values of $d$ and $I$ at the end of that execution. Thus, we are assuming that (12) and (13) hold for $d=d^{\prime}$ and $I=I^{\prime}$. Now the selection of $s$ and the definition of $d_{s}^{\prime \prime}$ ensure that

$$
\max _{j \in J} d_{s}^{\prime \prime} a_{s j} d_{j}^{\prime \prime}=\alpha_{k}=r_{s}
$$

As $d_{j}^{\prime \prime}=d_{j}^{\prime}$ for $j \in J \backslash\{s\}$ and as the specialization of (12) with $d=d^{\prime}$ to $i=s$ ensures that we have

$$
d_{s}^{\prime} a_{s j} d_{j}^{\prime} \leqq r_{s} \text { for all } j \in J,
$$

we conclude that $d_{s}^{\prime \prime} \geqq d_{s}^{\prime}$. As the remaining coordinates of $d$ are unchanged, it follows that $d^{\prime \prime} \geqq d^{\prime}$.

We next establish (12) and (13) with $d=d^{\prime \prime}$ and $I=I^{\prime \prime}$. First, if $i \in J \backslash\{s\}$ and $j \in$ $J \backslash\{s\}$, then $d_{i}^{\prime \prime} a_{i j} d_{j}^{\prime \prime}=d_{i}^{\prime} a_{i j} d_{j}^{\prime} \leqq r_{s}$. Also, (14) ensures that $d_{s}^{\prime \prime} a_{s j} d_{j}^{\prime \prime} \leqq r_{s}$ for all $j \in J$. Next, for $i \in J$ we have from the symmetry of $A$, from (14), and from the fact that $r_{s}=$ 
$\alpha_{k}=\min \left\{r_{j} \mid j \in J\right\}$ that

$$
d_{i}^{\prime \prime} a_{i s} d_{s}^{\prime \prime}=d_{s}^{\prime \prime} a_{s i} d_{i}^{\prime \prime} \leqq r_{s} \leqq r_{i},
$$

completing the proof that (12) holds for $d=d^{\prime \prime}$. Further, as it is assumed that (13) holds for $d=d^{\prime}$ and $I=I^{\prime}$, as $d^{\prime \prime} \geqq d^{\prime}$, and as we have seen that (12) holds for $d=d^{\prime \prime}$, we conclude that (13) is also valid with $d=d^{\prime \prime}$ and $I=I^{\prime}$. This fact combines with (14) to show that

$$
\max _{j \in J} d_{i}^{\prime \prime} a_{i j} d_{j}^{\prime \prime}=r_{i} \quad \text { for } i \in J \backslash I^{\prime \prime}=\left(J \backslash I^{\prime}\right) \cup\{s\} .
$$

That is, (13) holds for $d=d^{\prime \prime}$ and $I=I^{\prime \prime}$.

It remains to show that (12) and (13) hold upon each entrance of Step 2 from Step 1. This fact is obvious for the first entrance of Step 2 from Step 1 because then $J=I$; hence, (12) follows from (9) and (13) is vacuous. Next, assume that (12) and (13) hold for the $k$ th entrance of Step 2 from Step 1 and consider the $(k+1)$ st entrance, assuming that Step 1 leads to Step 2 rather than to termination. Our earlier arguments show that (12) and (13) will stay valid throughout consecutive iterations of Step 2; hence, they will hold at the $(k+1)$ st entrance into Step 1. Let $d^{\prime}, J^{\prime}$, and $I^{\prime}=\varnothing$ be the values of $d$, $J$, and $I$ upon the $(k+1)$ st entrance into Step 1 , and let $d^{\prime \prime}, J^{\prime \prime}$, and $I^{\prime \prime}$ be the updated values of $d, J$, and $I$ after the $(k+1)$ st execution of Step 1. In particular, $J^{\prime \prime} \backslash I^{\prime \prime}=$ $J^{\prime} \backslash I^{\prime}=J^{\prime}$, and (12) and (13) hold for $d=d^{\prime}, I=I^{\prime}$, and $J=J^{\prime}$. Further, as $d_{j}^{\prime \prime}=d_{j}^{\prime}$ for $j \in J^{\prime \prime} \backslash I^{\prime \prime}=J^{\prime}$, we have from the validity of (13) for $d=d^{\prime}, J=J^{\prime}$, and $I=I^{\prime}$ that for $i \in J^{\prime \prime} \backslash I^{\prime \prime}=J^{\prime} \backslash I^{\prime}$

$$
\begin{aligned}
\max _{j \in J^{\prime \prime}} d_{i}^{\prime \prime} a_{i j} d_{j}^{\prime \prime} & =\max \left\{\max _{j \in J^{\prime}} d_{i}^{\prime} a_{i j} d_{j}^{\prime}, \max _{j \in I^{\prime \prime}} d_{i}^{\prime \prime} a_{i j} d_{j}^{\prime \prime}\right\} \\
& =\max \left\{r_{i}, \max _{j \in I^{\prime \prime}} d_{i}^{\prime \prime} a_{i j} d_{j}^{\prime \prime}\right\} .
\end{aligned}
$$

Now, for $j \in I^{\prime \prime}, r_{j}=\alpha_{k+1}=\min \left\{r_{j} \mid j \in J^{\prime \prime}\right\}$; hence, the symmetry of $A$ and (9) with $d=d^{\prime \prime}, J=J^{\prime \prime}$, and $I=I^{\prime \prime}$ imply that for $j \in I^{\prime}$

$$
d_{i}^{\prime \prime} a_{i j} d_{j}^{\prime \prime}=d_{j}^{\prime \prime} a_{j i} d_{i}^{\prime \prime} \leqq \alpha_{k+1} \leqq \alpha_{k}=r_{i} .
$$

Combining (16) and (17) we conclude that

$$
\max _{j \in J^{\prime \prime}} d_{i}^{\prime \prime} a_{i j} d_{j}^{\prime \prime}=r_{i} \quad \text { for all } i \in J^{\prime \prime} \backslash I^{\prime \prime} .
$$

That is, (13) holds for $d=d^{\prime \prime}, J=J^{\prime \prime}$, and $I=I^{\prime \prime}$. This fact and (9) ensure that (12) holds as well. Thus, both (12) and (13) hold at the end of $(k+1)$ st execution of Step 1 and therefore at the entrance to Step 2.

THEOREM 7. If the Max Symmetric Scaling Algorithm is executed with input $A \in$ $\Re^{n \times n}$ and $r \in \Re^{n}$, then either the algorithm terminates in Step 3 with a positive diagonal matrix $D$ such that $D A D \in \Pi(r)$ or the algorithm terminates in Step 1 with $J \subseteq\langle n\rangle$ and $i \in J$ such that $A_{J J}$ is an r-upper principal submatrix of $A$ with $A_{i J}=0$. The algorithm requires $O(n \ln n+p)$ comparisons, $O(p)$ multiplications and divisions, and $O(q)$ square root calculations, where $p$ and $q$ are, respectively, the number of nonzero elements and the number of nonzero diagonal elements of the matrix $A$.

Proof. The algorithm must terminate as Step 1 is executed at most $m$ times, and each execution of Step 2 reduces $|I|$. If the algorithm terminates in Step 1, then $A_{i J}$ is a zero row of the $r$-upper principal submatrix $A_{J J}$. It follows from Lemma 6 that each time Step 3 is executed, we have $(D A D)_{J J} \in \Pi\left(r_{J}\right)$ because $I=\varnothing$ when Step 3 is exe- 
cuted. Therefore, if the algorithm terminates in Step 3, then $J=\langle n\rangle$ and $(D A D)_{J J}=$ $D A D \in \Pi(r)$.

We finally determine the complexity of the algorithm. With $O(n \ln n)$ comparisons, we can sort the values $\left\{r_{i} \mid i \in\langle n\rangle\right\}$ as required in Step 0. In total, Step 1 can be performed with $p$ comparisons and $p$ divisions, and Step 2 can be performed with $p-n$ comparisons, $p-q$ multiplications, $p$ divisions, and $q$ square root calculations.

The following example shows that the square root calculations cannot, in general, be eliminated. Let

$$
A=[1] \in \Re^{1 \times 1} \text { and } \quad r=(2) \in \Re^{1} \text {. }
$$

Then the only scaling $D A D$ of $A$ that is in $\Pi(r)$ has $D=\sqrt{2}$. If the diagonal elements of $A$ are all zero, then because the square-root operation in (9) can be omitted, the algorithm can be executed over any linearly ordered group (multiplicative) Abelian group with zero, that is, a group $G$ together with an element 0 such that $a 0=0=0 a$ for any $a \in G$. In particular, if the underlying matrix is nonnegative, then the output elements will be in any subgroup that contains the input elements. The above example shows that this conclusion need not hold when $A$ has nonzero diagonal elements.

We note that a diagonal element $a_{i i}$ is considered twice in the course of an execution of the algorithm. If $r_{i}=\alpha_{k}$, we have $a_{i i} \rightarrow a_{i i} d_{i}^{2}$ in the $k$ th execution of Step 1, and then in one of the following executions of Step 2, $\sqrt{\alpha_{k} / a_{i i}}=\sqrt{r_{i} / a_{i i}}$ is determined and is compared with other numbers to update $d_{i}$. Thus, the square rooting can be avoided if each original $a_{i i}$ is the product of $r_{i}$ and the square of a known number. Consequently, the square rooting can be avoided in the "decision problem" where one determines whether or not there exists a scaling corresponding to a given vector $r$ and matrices in a given pattern $P$. This is achieved by testing any matrix $A$ in $\Pi(P)$ with $a_{i i}=r_{i}$ for all $i$ with $P_{i i} \neq 0$.

6. Equivalence scaling. We apply our results for max symmetric scaling to max equivalence scaling.

For strictly positive $r=\left(r_{1}, \ldots, r_{m}\right)^{T} \in \Re^{m}$ and $c=\left(c_{1}, \ldots, c_{n}\right)^{T} \in \Re^{n}$, let $M(r, c)$ denote the set of all $m \times n$ nonnegative matrices $A=\left[a_{i j}\right]$ such that

$$
\max _{j \in\langle n\rangle} a_{i j}=r_{i} \quad \text { for } i \in\langle m\rangle \text { and } \max _{i \in\langle m\rangle} a_{i j}=c_{j} \quad \text { for } j \in\langle n\rangle .
$$

In the following theorem we characterize the existence of a solution for the max equivalence scaling by reducing it to max symmetric scaling.

THEOREM 8. Let $P$ be an $m \times n$ pattern matrix, and let $r \in \Re^{m}$ and $c \in \Re^{n}$ be strictly positive. Then the following are equivalent:

(i) Some $A \in \Pi(P)$ has an equivalence scaling in $M(r, c)$.

(ii) Each $A \in \Pi(P)$ has an equivalence scaling in $M(r, c)$.

(iii) The set $\Pi(P) \cap M(r, c)$ is nonempty.

(iv) The set $\Pi\left(P^{\prime}\right) \cap M(r, c)$ is nonempty for some pattern matrix $P^{\prime} \leqq P$.

(v) The vectors $r$ and $c$ satisfy

$$
\max _{i \in\langle m\rangle} r_{i}=\max _{j \in\langle n\rangle} c_{j}
$$

furthermore, if $P_{I J}=0$ for subsets $I \subseteq\langle m\rangle$ and $J \subseteq\langle n\rangle$, then

$$
\max _{i \in I} r_{i} \leqq \max _{j \in J^{c}} c_{j} \text { and } \max _{j \in J} c_{j} \leqq \max _{i \in I^{c}} r_{i} \text {. }
$$


(vi) The following conditions hold:

(a) The vectors $r$ and c satisfy (19).

(b) If $P_{i J}=0$ for $i \in\langle m\rangle$ and $J \subseteq\langle n\rangle$, then

$$
r_{i} \leqq \max _{j \in J^{c}} c_{j}
$$

(c) If $P_{I j}=0$ for $I \subseteq\langle m\rangle$ and $j \in\langle n\rangle$, then

$$
c_{j} \leqq \max _{i \in I^{c}} r_{i} .
$$

(vii) The vectors $r$ and $c$ satisfy (19), and for all $\alpha \in \Re$ and subsets $I=$ lev $(\alpha, r) \subseteq\langle m\rangle$ and $J=\operatorname{lev}(\alpha, c) \subseteq\langle n\rangle$, if $I, J \neq \varnothing$, then the submatrix $P_{I J}$ contains neither a zero row nor a zero column.

Proof. The implications (i) $\Rightarrow$ (ii) $\Rightarrow$ (iii) $\Rightarrow$ (iv) follow from the arguments used to establish the corresponding implications in Theorem 5 . Then

(iv) $\Rightarrow$ (v): Suppose that $A \in \Pi\left(P^{\prime}\right) \cap M(r, c)$ for some pattern matrix $P^{\prime} \leqq P$.

$$
\max _{i \in\langle m\rangle} r_{i}=\max _{i \in\langle m\rangle} \max _{j \in\langle n\rangle} a_{i j}=\max _{j \in\langle n\rangle} \max _{i \in\langle m\rangle} a_{i j}=\max _{j \in\langle n\rangle} c_{j} .
$$

Furthermore, if $P_{I J}=0$ for some $I \subseteq\langle m\rangle$ and $J \subseteq\langle n\rangle$, then

$$
\max _{i \in I} r_{i}=\max _{i \in I} \max _{j \in J^{c}} a_{i j}=\max _{j \in J^{c}} \max _{i \in I} a_{i j} \leqq \max _{j \in J^{c}} c_{j} .
$$

A symmetric argument proves the second inequality in (20).

(v) $\Rightarrow$ (vi): This implication is trite because parts (b) and (c) of condition (vi) are the specializations of the second part of condition ( $v$ ) for the cases of $I=i$ and $J=j$, respectively.

(vi) $\Rightarrow$ (vii): Suppose that for some $\alpha \in \Re, I=\operatorname{lev}(\alpha, r) \neq \varnothing$ and $J=$ lev $(\alpha, c) \neq \varnothing$. It follows that

$$
r_{i} \geqq \alpha>\max _{j \in J^{c}} c_{j} \quad \text { for } i \in I
$$

and

$$
c_{j} \geqq \alpha>\max _{i \in I^{c}} r_{i} \quad \text { for } j \in J .
$$

Therefore, if $P_{I J}$ has a zero row or a zero column, we get a violation of parts (b) or (c), respectively, of condition (vi). The implication (vi) $\Rightarrow$ (vii) now follows because the first assertion of (vii) is the same as part a of (vi).

(vii) $\Rightarrow$ (i): For an $m \times n$ nonnegative matrix $A$, define the $(m+n) \times(m+n)$ matrix $A^{\prime}$ and the vector $r^{\prime} \in \Re^{m+n}$ by

$$
A^{\prime}=\left(\begin{array}{c|c}
0 & A \\
\hline A^{T} & 0
\end{array}\right) \quad \text { and } \quad r^{\prime}=\left(\frac{r}{c}\right) \text {. }
$$

It is straightforward to show that max symmetric scaling with input $A^{\prime}$ and $r^{\prime}$ has a solution $D^{\prime}=\operatorname{diag}\left(d_{1}^{\prime}, d_{2}^{\prime}, \ldots, d_{m+n}^{\prime}\right)$ if and only if max equivalence scaling for $A, r$, and $c$ has a solution $D=\operatorname{diag}\left(d_{1}^{\prime}, d_{2}^{\prime}, \ldots, d_{m}^{\prime}\right)$ and $E=\operatorname{diag}\left(d_{m+1}^{\prime}, \ldots, d_{m+n}^{\prime}\right)$. We conclude that condition (i) of Theorem 8 is equivalent to condition (i) of Theorem 5 applied to $A^{\prime}$ and $r^{\prime}$. It is straightforward to show that condition (vii) of Theorem 8 is 
equivalent to condition (vii) of Theorem 5 when applied to $A^{\prime}$ and $r^{\prime}$. Therefore, the equivalence of (i) and (vii) follows from Theorem 5.

We observe that the reduction of max equivalence scaling to max symmetric scaling in the proof of Theorem 8 shows that max equivalence scaling can be solved by the Max Symmetric Scaling Algorithm. Moreover, the diagonal elements of the matrix $A^{\prime}$ defined in (21) are all zero. Therefore, it follows from the complexity analysis at the end of $\S 5$ that max equivalence scaling can be solved using $O((n+m) \ln (n+m)+p)$ comparisons and $O(p)$ multiplications and divisions, where $p$ is the number of nonzero elements of the matrix $A$. Further, we observe that max equivalence scaling can be solved over any linearly ordered Abelian group with zero. The example given in $\S 5$ shows that in general max symmetric scaling does not have this property. Also, sum equivalence scaling does not have this property. For example, let

$$
A=\left(\begin{array}{ll}
1 & 2 \\
1 & 1
\end{array}\right) \text { and } r=c=\left(\begin{array}{l}
1 \\
1
\end{array}\right) .
$$

The only equivalence scaling of $A$ with row and column sums all equality 1 is the matrix

$$
B=\left(\begin{array}{cc}
\sqrt{2}-1 & 2-\sqrt{2} \\
2-\sqrt{2} & \sqrt{2}-1
\end{array}\right)=\left(\begin{array}{cc}
1-2^{-1} \sqrt{2} & 0 \\
0 & \sqrt{2}-1
\end{array}\right) A\left(\begin{array}{cc}
\sqrt{2} & 0 \\
0 & 1
\end{array}\right) .
$$

We note that when a solution to max equivalence scaling exists, it need not be unique. For example, let

$$
A=\left(\begin{array}{ll}
4 & 1 \\
2 & 4
\end{array}\right) \text { and } r=c=\left(\begin{array}{l}
4 \\
4
\end{array}\right) \text {. }
$$

Then the general equivalence scaling $B$ of $A$ that is in $M(r, c)$ is given by

$$
B=\left(\begin{array}{cc}
1 & 0 \\
0 & \alpha
\end{array}\right) A\left(\begin{array}{cc}
1 & 0 \\
0 & \alpha^{-1}
\end{array}\right)=\left(\begin{array}{cc}
4 & \alpha^{-1} \\
2 \alpha & 4
\end{array}\right) \text {, }
$$

where $\frac{1}{4} \leqq \alpha \leqq 2$. By contrast, the corresponding equivalence in sum equivalence scaling is unique.

The results about max equivalence scaling were derived from results about max symmetric scaling. Historically, a reverse logic has been applied in the sum case as results about sum equivalence scaling are used to establish results about sum symmetric scaling (see Brualdi [2] and Csima and Datta [7]). The latter arguments use uniqueness (up to multiplicative scalar) of diagonal matrices $D$ and $E$ for which $D A E$ has prescribed row sum vector $r$ and column sum vector $c$. Hence, if $A$ is symmetric and $r=c$, the fact that $D A E$ and $E A^{T} D=E A D$ have the same row and column sums can be used to argue that (with proper normalization) $D=E$. But, as we have seen above, no such uniqueness results are available in the max case.

7. Graphs. We observe that max symmetric scaling and the corresponding solvability theorem have an equivalent undirected graph statement. An (undirected) graph is an ordered pair $G=(V, E)$, where $V$ is a finite set of vertices and $E$ is a set of edges composed of unordered pairs of vertices. Given such a graph $G=(V, E)$ and a vertex $v \in V$, we let $N(v)$ denote the set of neighbors of $v$, i.e., $N(v) \equiv\{u \in V:\{u, v\} \in E\}$ : In particular, we say that $v$ is isolated if $N(v)=\phi$. Note that by definition a graph may contain loops but may not contain repeated edges. For subsets $S, T \subseteq V$, we use $[S, T]$ to denote the set of edges $\{u, v\} \in E$ with $u \in S$ and $v \in T$ or $u \in T$ and $v \in S$. 
A weight function for a graph $G=(V, E)$ is a real-valued function $f$ defined on the edges $E$. For convenience, in this case we write $f_{u v}$ for $f(\{u, v\})$. A weighted graph is a triple $(V, E, f)$, where $(V, E)$ is a graph and $f$ is a weight function for $G$. A potential for $G$ is a real-valued function defined on the vertices $V$. For a nonempty subset $W$ of $V$, we define the subgraph induced by $W$ to be the graph $\left(W, E^{\prime}\right)$, where $E^{\prime}$ contains all edges of $E$ of the form $e=\{u, v\}$ for vertices $u, v \in W$.

Next, we define a mapping $\Phi$ from the set of symmetric nonnegative matrices to the set of weighted graphs. For an $n \times n$ symmetric nonnegative matrix $A=\left[a_{i j}\right]$, we define the mapping $\Phi$ by

$$
A \stackrel{\Phi}{\rightarrow}(V, E, f)
$$

where

$$
\begin{aligned}
V & =\langle n\rangle, \\
E & =\left\{\{i, j\} \mid a_{i j}>0\right\}, \quad \text { and } \\
f_{i j} & =\ln a_{i j} \text { for }\{i, j\} \in E .
\end{aligned}
$$

It is easy to see that $\Phi$ is a bijection between the set of nonnegative symmetric matrices and the set of weighted graphs.

We state the following lemma without proof.

LEMMA 9. Let $A$ be an $n \times n$ symmetric nonnegative matrix, and let $(V, E, f)$ be the corresponding weighted graph under the mapping $\Phi$ in (22). Let $r \in \Re^{n}$ be strictly positive, and let $s$ be the potential defined by $s_{i}=\ln r_{i}$ for $i \in\langle n\rangle$. Then the following are equivalent:

(i) There exists a positive diagonal matrix $D=\operatorname{diag}\left(d_{1}, d_{2}, \ldots, d_{n}\right)$ such that $D A D \in M(r)$.

(ii) There exists a potential $p$ such that

$$
\max _{u \in N(v)}\left\{p_{u}+f_{u v}+p_{v}\right\}=s_{v} \quad \text { for } v \in V .
$$

Furthermore, $D$ and $p$ are related by $p_{u}=\ln d_{u}$ for $u \in\langle n\rangle=V$.

The next theorem follows directly from Theorem 5 and the correspondence between matrices and graphs described in Lemma 9.

THEOREM 10. Let $G=(V, E)$ be a graph, and let $s$ be a potential for $G$. Then the following are equivalent:

(i) For every weight function ffor $G$ there exists a potential p satisfying (23).

(ii) For some weight function f for $G$ there exists a potential p satisfying (23).

(iii) There exists some weight function f for $G$ such that

$$
\max _{u \in N(v)} f_{u v}=s_{v} \quad \text { for } v \in V .
$$

(iv) There exists $E^{\prime} \subseteq E$ and some weight function $f: E^{\prime} \mapsto \Re$ such that

$$
\max _{u \in N^{\prime}(v)} f_{u v}=s_{v} \quad \text { for } v \in V,
$$

where $N^{\prime}(v)=\left\{u \in V:\{u, v\} \in E^{\prime}\right\}$.

(v) If $[S, T]=\varnothing$ for $S, T \subseteq V$, then

$$
\max _{v \in S} S_{v} \leqq \max _{v \in T^{c}} s_{v} .
$$


(vi) If $[S, T]=\varnothing$ for $S, T \subseteq V$, then

$$
\max _{v \in S \cap T} s_{v} \leqq \max _{v \in(S \cup T)^{c}} s_{v}
$$

(vii) If $\{S, T, U\}$ is any partition of $V$ such that $[S, S \cup T]=\varnothing$, then

$$
\max _{v \in S} s_{v} \leqq \max _{v \in U} s_{v} .
$$

(viii) Let $W \subseteq V$. If $v \in W$ is an isolated vertex of the subgraph induced by $W$, then

$$
s_{v} \leqq \max _{u \in W^{c}} s_{u} .
$$

(ix) For every $\alpha \in \Re$, the subgraph of $G$ induced by the level set lev $(s, \alpha)$ has no isolated vertex.

We observe that Theorem 8 also has an equivalent graph formulation in terms of bipartite graphs. We have omitted the details because they are straightforward.

8. $p$ th power scaling. For $0 \leqq p \leqq \infty$ and $x \in \Re^{n}$, we define the $l_{p}$ norm of $x$ by $\|x\|_{p}$. We consider the problem of determining a symmetric scaling of a given nonnegative symmetric matrix such that the rows of the resulting matrix have prescribed $l_{p}$ norms. Of course, the cases of $p=1$ and $p=\infty$ reduce to sum and max symmetric scaling, respectively. Here, we show that the cases of $0<p<\infty$ can be reduced easily to the case of $p=1$.

For an $m \times n$ nonnegative matrix $A$ and $0<p<\infty$, the $p$ th Hadamard power of $A$, written $A^{(p)}$, is the matrix whose $i j$ th entry is $\left(a_{i j}\right)^{p}$. Let $A_{i}$ denote the $i$ th row of the matrix $A$. For a strictly positive vector $r \in \Re^{n}$ and $0<p \leqq \infty$, let $S^{p}(r)$ denote the set of all $n \times n$ nonnegative symmetric matrices $B$ such that $\left\|B_{i}\right\|_{p}=r_{i}$ for each $i \in\langle n\rangle$.

It is easily seen that $B \in S^{p}(r)$ if and only if $B^{(p)} \in S\left(r^{(p)}\right)$. Moreover, $B=D A D$ if and only if $B^{(p)}=D^{(p)} A^{(p)} D^{(p)}$. Thus, as an immediate consequence of Theorem 1, we obtain the following theorem.

THEOREM 11 ( $l_{p}$-symmetric scaling). Let $P$ be an $n \times n$ symmetric pattern matrix, let $r \in \Re^{n}$ be strictly positive, and let $p \in \Re$ with $0<p<\infty$. Then the following are equivalent:

(i) Each symmetric $A \in \Pi(P)$ has a symmetric scaling $B \in S^{p}(r)$.

(ii) Some symmetric $A \in \Pi(P)$ has a symmetric scaling $B \in S^{p}(r)$.

(iii) The set $\Pi(P) \cap S^{p}(r)$ is nonempty.

(iv) If $P_{I J}=0$ for $I, J \subseteq\langle n\rangle$, then $\left\|r_{I}\right\|_{p} \leqq\left\|r_{y c}\right\|_{p}$ with equality holding if and only if $P_{I^{c} J^{c}}=0$.

Conditions (v) and (vi) of Theorem 1 can also be extended in the obvious way. Further, analogous results can also be derived for the cases of $-\infty \leqq p<0$.

\section{REFERENCES}

[1] L. M. BREGMaN, Proof of the convergence of Sheleikhovskii's method for a problem with transportation constraints, U.S.S.R. Comput. Math. and Math. Phys. 1 (1967), pp. 191-204.

[2] R. A. BRualdi, Convex sets of non-negative matrices, Canad. J. Math., 20 (1968), pp. 144-157.

[3] - The DAD theorem for arbitrary row sums, Proc. Amer. Math. Soc., 45 (1974), pp. 189-194.

[4] Combinatorial properties of symmetric non-negative matrices, in Colloquio Internazionale sulle Teorie Combinatorie, Accademia Nazional de Lincei, Rome, 1976, pp. 99-120.

[5] R. A. BRuAldi, S. PARTER, AND H. SCHNEIDER, The diagonal equivalence of a nonnegative matrix to a stochastic matrix, J. Math. Anal. Appl., 16 (1966), pp. 31-50. 
[6] V. ChVÁtal, Linear Programming. W. H. Freeman and Company, New York, 1983.

[7] J. CSIMA AND B. N. DATTA, The DAD theorem for symmetric non-negative matrices, J. Combin. Theory Ser. A, 12 (1972), pp. 147-152.

[8] D. Gale, A theorem on flows in networks, Pacific J. Math., 7 (1957), pp. 1073-1082.

[9] A. J. HofFMAN, Some recent applications of the theory of linear inequalities to extremal combinatorial analysis, Combinatorial Analysis, in R. Bellman and M. Hall, Jr., eds., American Mathematical Society, Providence, RI, 1960, pp. 113-127.

[10] J. KRUITHOF, Telefoonverkeersrekening, De Ingenieur, 3 (1937), pp. 15-25.

[11] A. W. MARSHALL AND I. OLKIN, Scaling of matrices to achieve specified row and column sums, Numer. Math., 12 (1968), pp. 83-90.

[12] M. V. MENON, Matrix links, an extremisation problem and the reduction of a nonnegative matrix to one with prescribed row and column sums, Canad. J. Math., 20 (1968), pp. 225-232.

[13] M. V. MENON AND H. SCHNEIDER, The spectrum of a nonlinear operator associated with a matrix, Linear Algebra Appl., 2 (1969), pp. 321-334.

[14] U. G. ROTHBLUM AND H. SCHNEIDER, Scaling of matrices which have prespecified row sums and column sums via optimization, Linear Algebra Appl., 114/115 (1989), pp. 737-764.

[15] U. G. Rothblum, H. SCHNEIDER, AND M. H. SCHNEIDER, Scaling matrices to prescribed row and column maxima, Rutgers Research Report, Rutgers University, New Brunswick, NJ, 1990.

[16] M. H. SCHNEIDER AND S. ZENIOS, A comparative study of algorithms for matrix balancing, Oper. Res., 38 (1990), pp. 439-455.

[17] R. SINKHORN, A relationship between arbitrary positive matrices and doubly stochastic matrices, Ann. Math. Statist., 35 (1964), pp. 876-879.

[18] R. SINKHORN AND P. KNOPP, Concerning nonnegative matrices and doubly stochastic matrices, Pacific J. Math., 212 (1967), pp. 343-348. 